\title{
-Supplementary Material- Negative Singlet-Triplet Excitation Energy Gap in Triangle-Shaped Molecular Emitters for Efficient Triplet Harvesting
}

\author{
J. Sanz-Rodrigo $^{a}$, G. Ricci ${ }^{b}$, \\ Y. Olivier ${ }^{b *}$, and J. C. Sancho-García ${ }^{a^{*}}$ \\ ${ }^{a}$ Department of Physical Chemistry, \\ University of Alicante, \\ E-03080 Alicante, Spain \\ ${ }^{b}$ Unité de Chimie Physique Théorique et Structurale \\ \& Laboratoire de Physique du Solid, \\ Namur Institute of Structured Matter, \\ Université de Namur, \\ B-5000 Namur, Belgium
}

December 14, 2020

*E-mail: yoann.olivier@unamur.be

${ }^{*}$ E-mail: jc.sancho@ua.es 


\section{Contents}

1 The spatial distribution of FOD densities $\quad$ S3

2 The case of 5T-a as a proof of concept $\quad$ S5

3 Benchmarking results for the 2T-a molecule $\quad$ S7

4 The correlation between $N_{F O D}$ and NEVPT2 electronic ex$\begin{array}{ll}\text { citation energies } & \text { S8 }\end{array}$

5 The overestimation of NEVPT2 electronic excitation energies by TDA-DFT $\quad \mathbf{S 1 0}$

6 The representation of frontier molecular orbitals $\quad \mathrm{S12}$

7 The role of double and higher excitations at the CASSCF level $\quad$ S16

$\begin{array}{lll}8 & \text { Difference density plots } & \text { S17 }\end{array}$

9 Relationships between $\Delta E_{S T}$ and the CT delocalization volume

10 Zero-field splitting and triplet exciton size 
1 The spatial distribution of FOD densities 


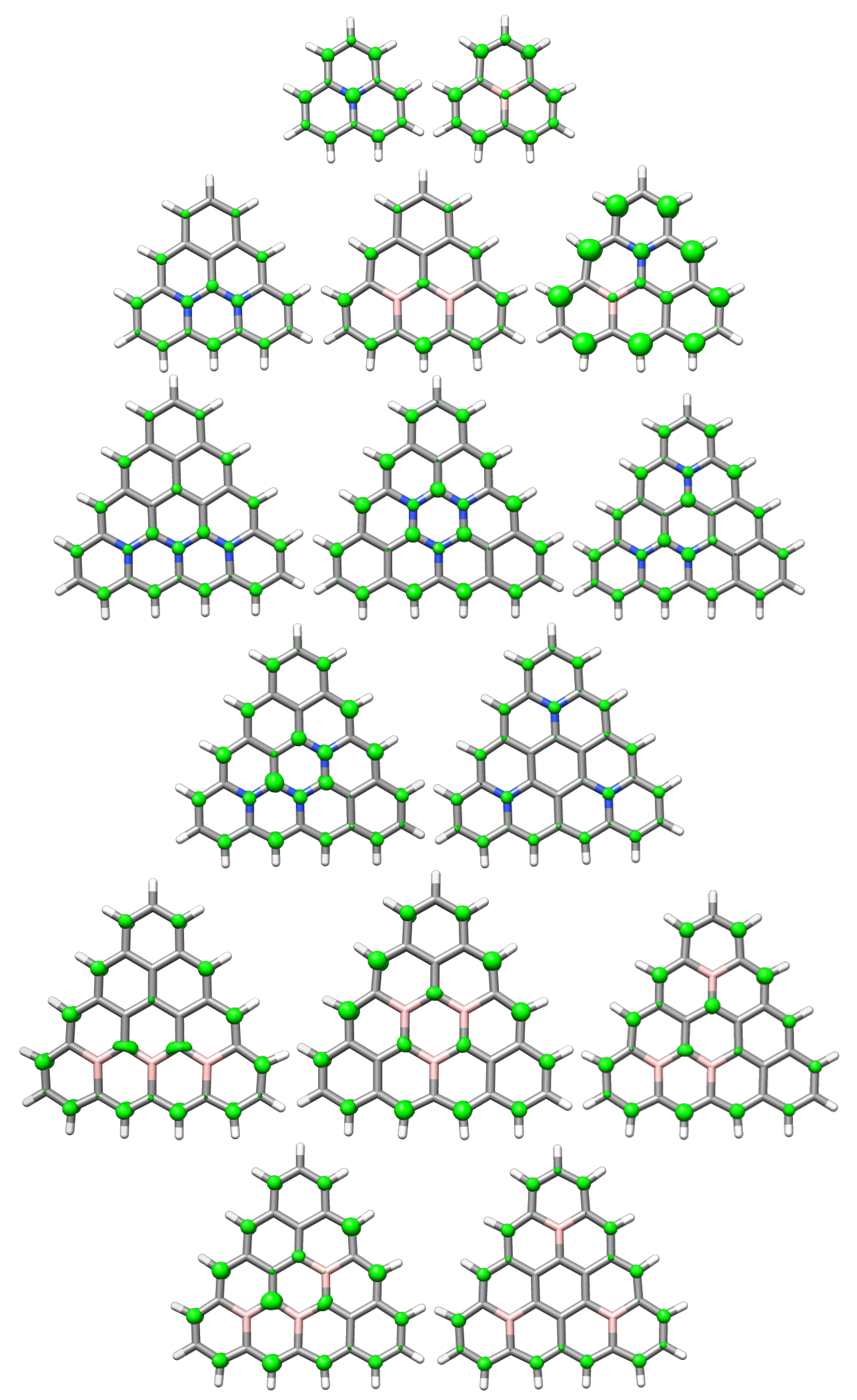

Figure S1: FOD density plots $\left(\sigma=0.005 \mathrm{e} / \mathrm{bohr}^{3}\right)$ obtained from the FTTPSS/def2-TZVP method for the whole set of studied compounds. From top to bottom and from left to right: 2T-a, 2T-b, 3T-a, 3T-b, 3T-c, 4T-a, $4 \mathrm{~T}-\mathrm{b}, 4 \mathrm{~T}-\mathrm{c}, 4 \mathrm{~T}-\mathrm{d}, 4 \mathrm{~T}-\mathrm{e}, 4 \mathrm{~T}-\mathrm{f}, 4 \mathrm{~T}-\mathrm{g}, 4 \mathrm{~T}-\mathrm{h}, 4 \mathrm{~T}-\mathrm{i}$, and $4 \mathrm{~T}-\mathrm{j}$. 
2 The case of 5T-a as a proof of concept 

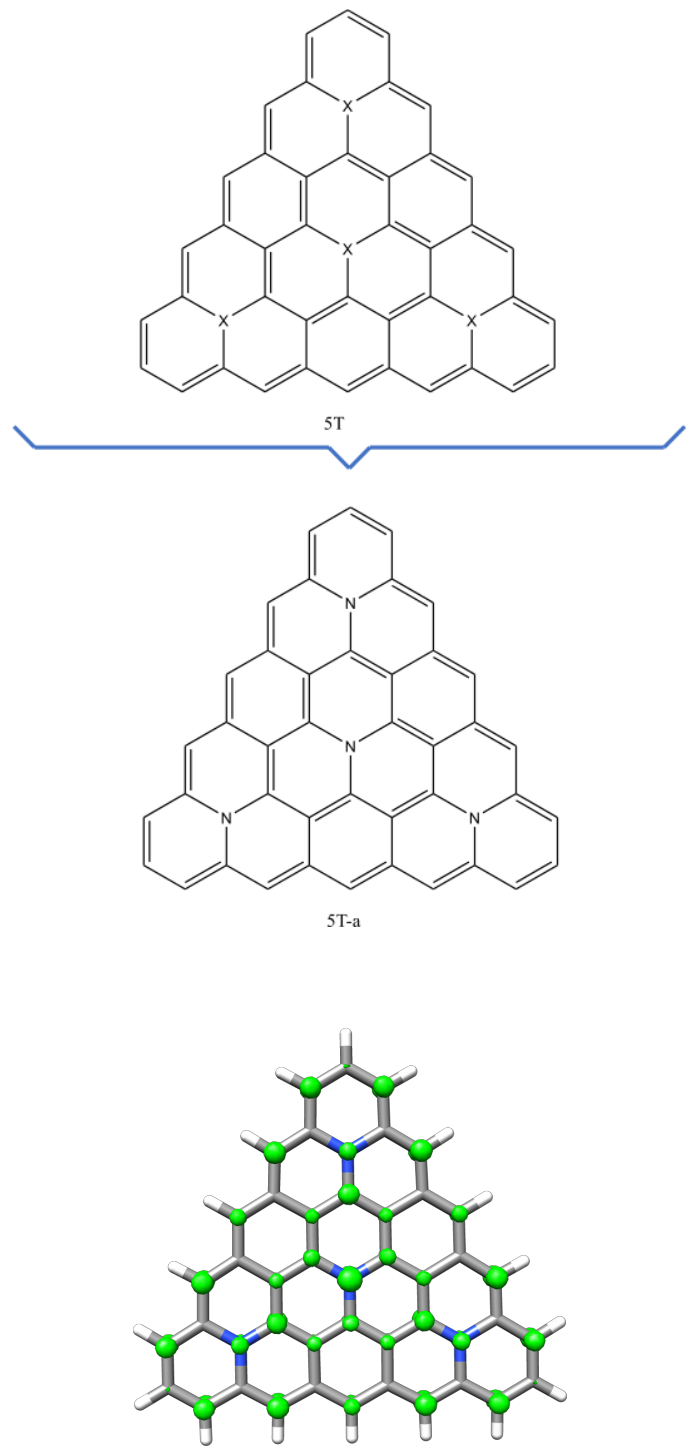

Figure S2: Chemical structure (top) and FOD density plot $(\sigma=0.005$ e/bohr ${ }^{3}$ ) obtained from the FT-TPSS/def2-TZVP method for the Nsubstituted $5 \mathrm{~T}$ system (5T-a) taken as example. 


\section{Benchmarking results for the 2T-a molecule}

Table S1: Energy magnitudes (in eV) calculated at different $a b$ initio levels for the 2T-a compound.

\begin{tabular}{|c|c|c|c|}
\hline Method & $S_{1} \leftarrow S_{0}$ & $T_{1} \leftarrow S_{0}$ & $\Delta E_{S T}$ \\
\hline 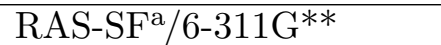 & 0.85 & 0.96 & -0.11 \\
\hline $\operatorname{ADC}(2)^{b} / c c-p V D Z$ & 1.04 & 1.20 & -0.16 \\
\hline EOM-CCSD ${ }^{\mathrm{b}} / \mathrm{cc}-\mathrm{pVDZ}$ & 1.09 & 1.19 & -0.10 \\
\hline SA-CASSCF/def2-SVP & 0.831 & 0.969 & -0.14 \\
\hline SA-CASSCF/def2-TZVP & 0.826 & 0.936 & -0.11 \\
\hline SA-CASSCF/def2-QZVP & 0.824 & 0.931 & -0.11 \\
\hline SC-NEVPT2/def2-SVP & 1.102 & 1.319 & -0.21 \\
\hline SC-NEVPT2/def2-TZVP & 1.107 & 1.259 & -0.15 \\
\hline SC-NEVPT2/def2-QZVP & 1.104 & 1.253 & -0.15 \\
\hline SCS-CC2/def2-TZVP & 1.11 & 1.33 & -0.22 \\
\hline Exp. ${ }^{\mathrm{c}}$ & 0.97 & 0.93 & 0.04 \\
\hline
\end{tabular}

a Taken from Ref. 1

b Taken from Ref. 2

c Taken from Ref. 3 
4 The correlation between $N_{F O D}$ and NEVPT2 electronic excitation energies 

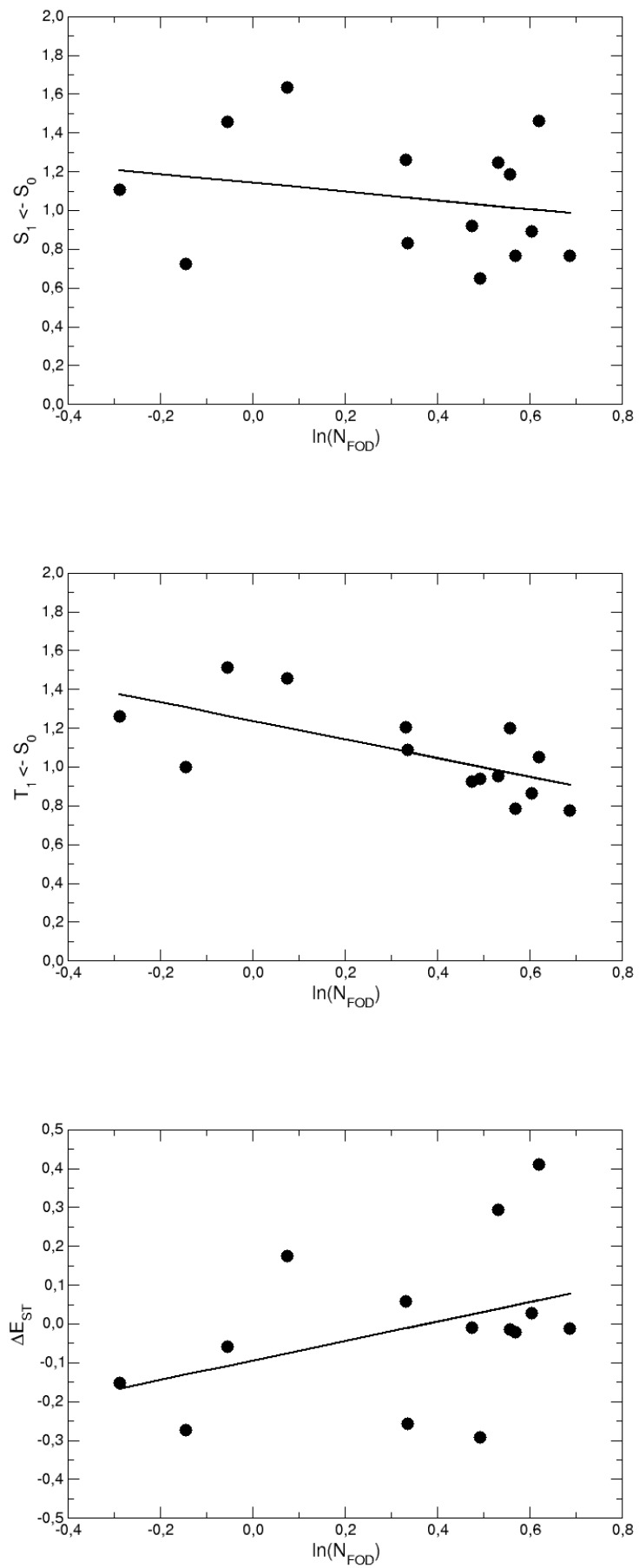

Figure S3: Relationships (from top to bottom) between $S_{1} \leftarrow S_{0}, T_{1} \leftarrow S_{0}$, and $\Delta E_{S T}$ (all calculated at the NEVPT2/def2-TZVP level, in eV) and $N_{F O D}$ values. 
5 The overestimation of NEVPT2 electronic excitation energies by TDA-DFT 

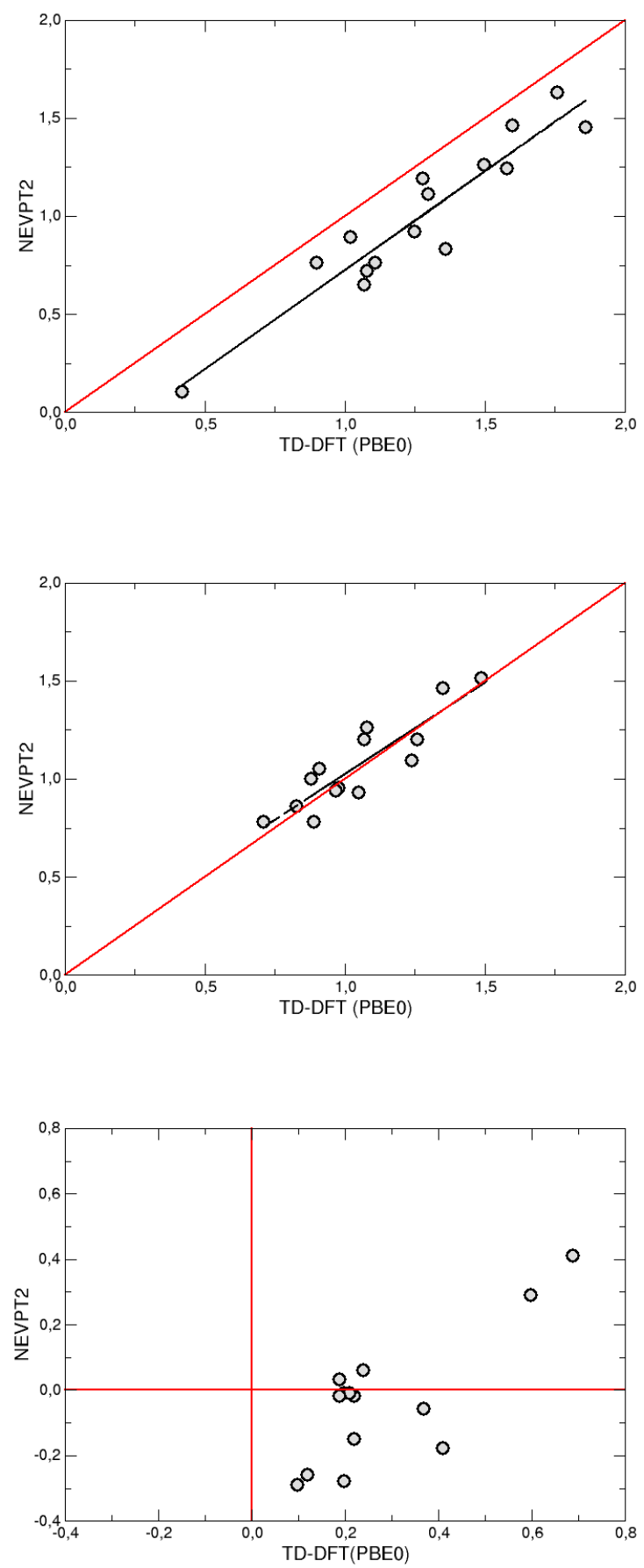

Figure S4: Comparison between NEVPT2/def2-TZVP and TDA-DFT (PBE0/def2-TZVP) methods (from top to bottom) for the calculated electronic excitation energies $S_{1} \leftarrow S_{0}$ (lineal regression: $y=-0.28522+$ $\left.1.0083 x ; r^{2}=0.941\right), T_{1} \leftarrow S_{0}$ (lineal regression: $y=0.08951+0.93521 x$; $\left.r^{2}=0.886\right)$, and $\Delta E_{S T}($ all in $\mathrm{eV})$. The red lines are a guide to the eye. 
6 The representation of frontier molecular orbitals 
HOMO-1

HOMO
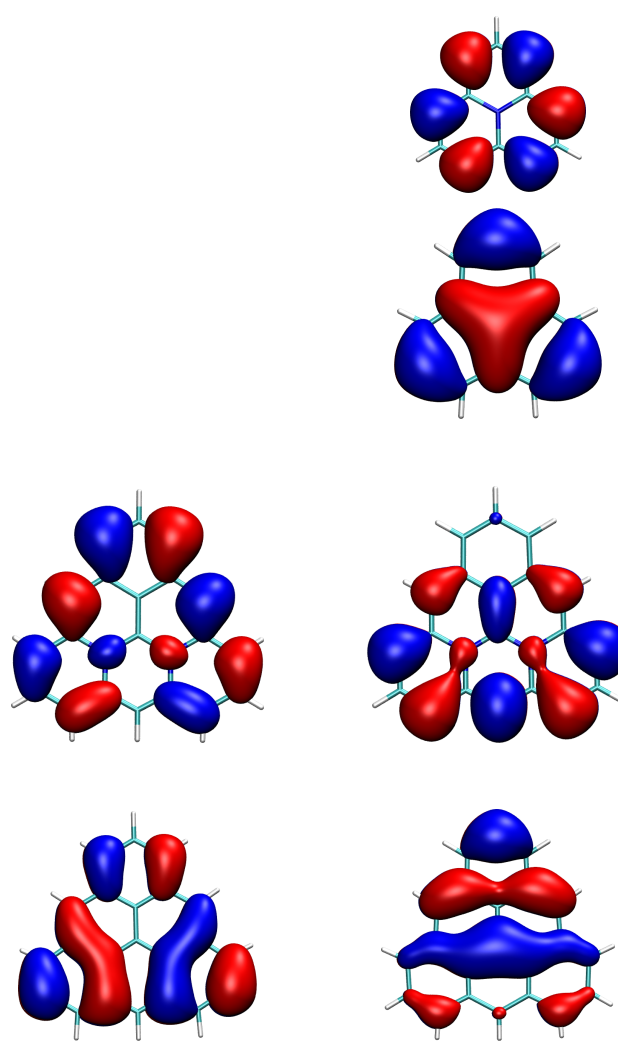

LUMO
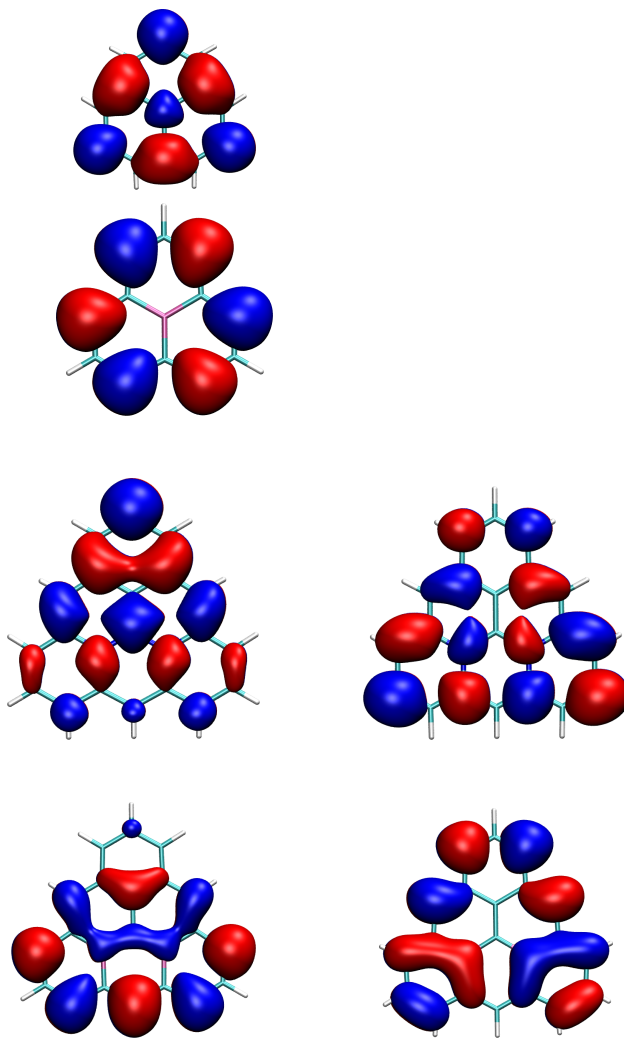
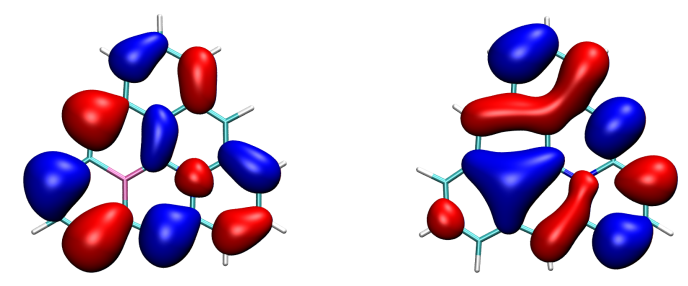

Figure S5: HOMO (left) and LUMO (right) molecular orbitals plots ( $\sigma=$ $0.02 \mathrm{e} / \mathrm{bohr}^{3}$ ) obtained at the HF/def2-TZVP level for the whole set of studied compounds. From top to bottom: 2T-a, 2T-b, 3T-a, 3T-b, and 3Tc. For $3 \mathrm{~T}-\mathrm{a}$ and $3 \mathrm{~T}-\mathrm{b}$ the HOMO-1 and LUMO+1 orbitals are also included since they are also involved in the corresponding $S_{1} \leftarrow S_{0}$ and $T_{1} \leftarrow S_{0}$ transitions. 

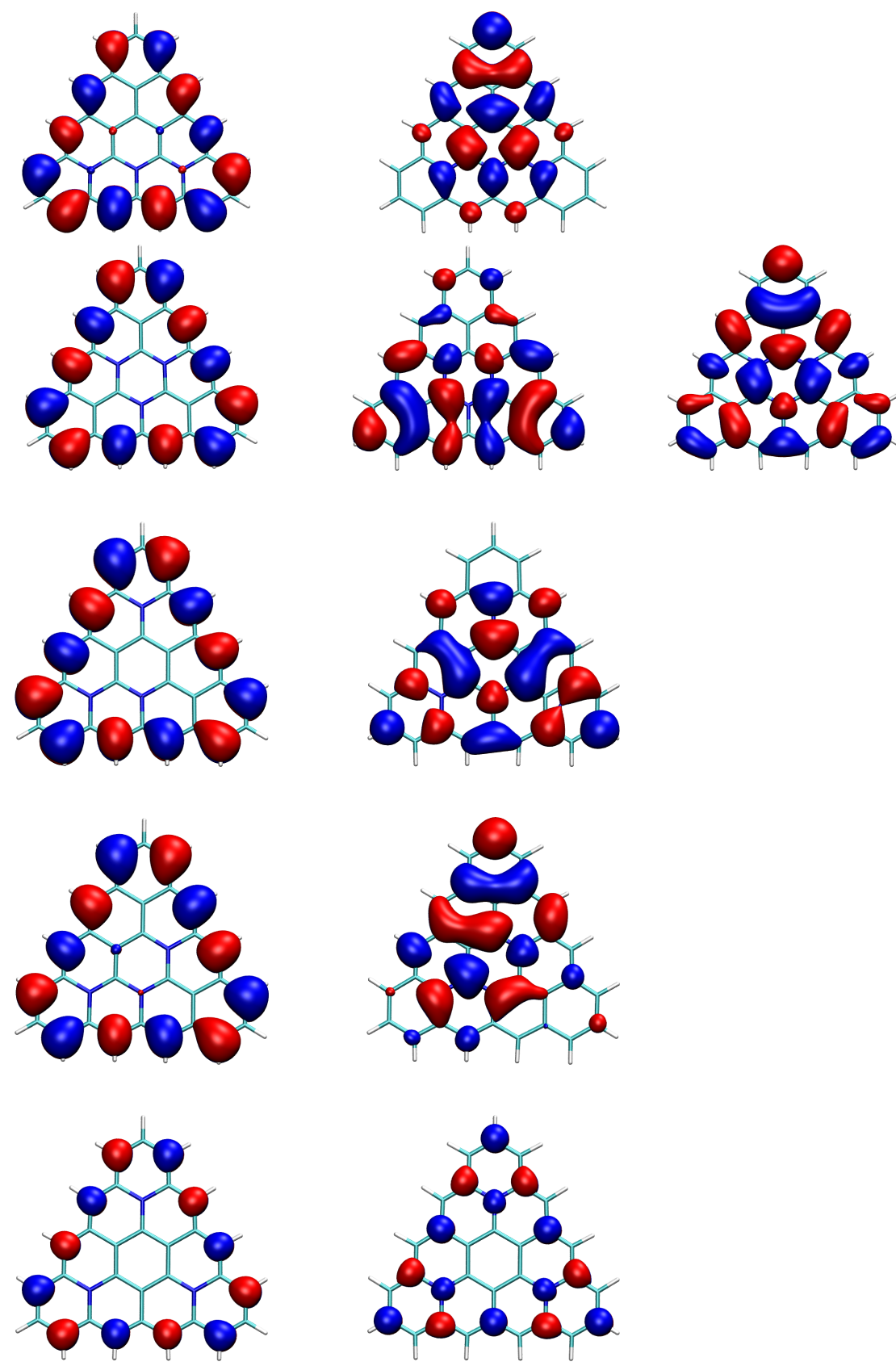

Figure S5 (cont.): HOMO (left) and LUMO (right) molecular orbitals plots $\left(\sigma=0.02 \mathrm{e} / \mathrm{bohr}^{3}\right)$ obtained at the HF/def2-TZVP level for the whole set of studied compounds. From top to bottom: 4T-a, 4T-b, 4T-c, 4T-d, and $4 \mathrm{~T}$-e. We also include the $\mathrm{LUMO}+1$ orbital for the $4 \mathrm{~T}-\mathrm{b}$ case due to its degeneracy with the LUMO. 
HOMO-1
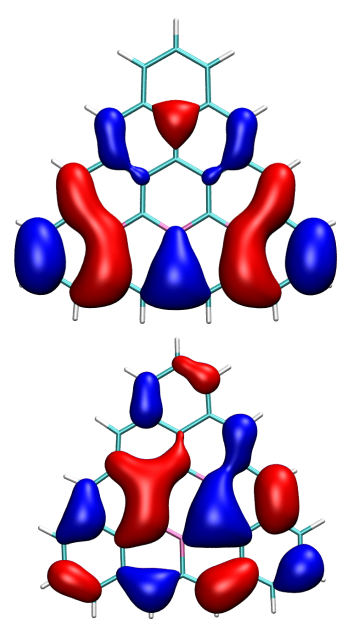

HOMO
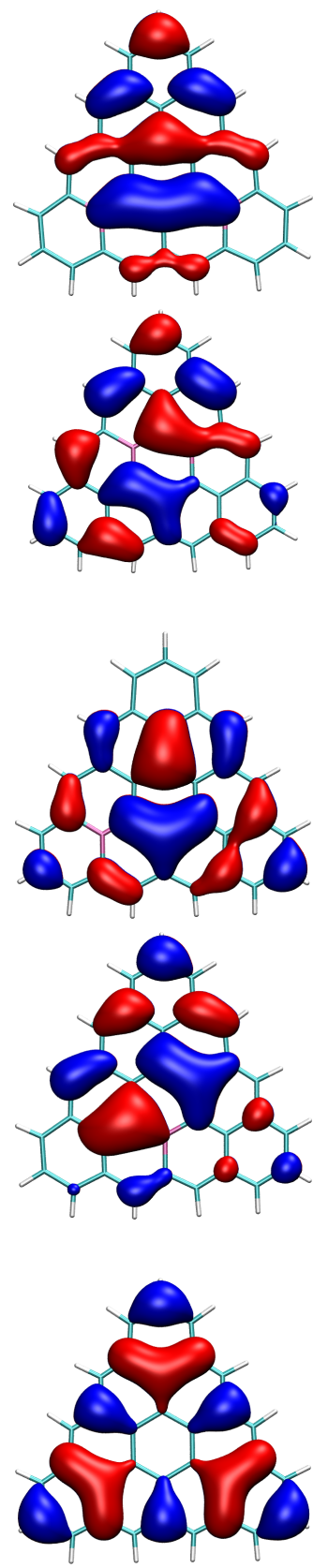

LUMO
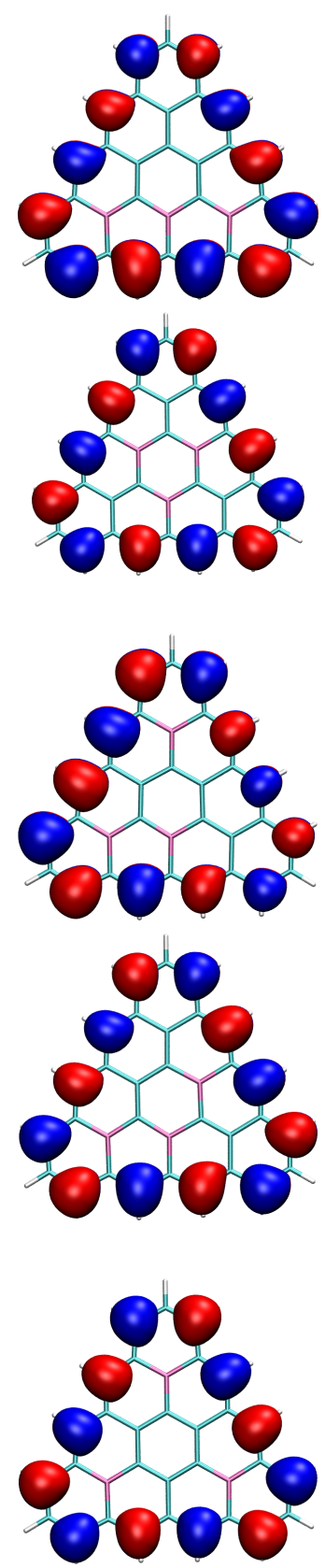

Figure S5 (cont.): HOMO (left) and LUMO (right) molecular orbitals plots $\left(\sigma=0.02 \mathrm{e} / \mathrm{bohr}^{3}\right)$ obtained at the HF/def2-TZVP level for the whole set of studied compounds. From top to bottom: 4T-f, 4T-g, 4T-h, 4T-i, and $4 \mathrm{~T}-\mathrm{j}$. We also include the HOMO-1 orbital for the $4 \mathrm{~T}-\mathrm{f}$ and $4 \mathrm{~T}-\mathrm{g}$ cases due to its degeneracy with the HOMO. 
7 The role of double and higher excitations at the CASSCF level

Table S2: $\Sigma_{i j \ldots}^{a b \ldots}$ values (in \%) for the $S_{0}, S_{1}$, and $T_{1}$ states of all the compounds.

\begin{tabular}{lrrr}
\multicolumn{4}{l}{$T_{1}$ states of all the compounds. } \\
\hline Compound & $\Sigma_{i j \ldots .}^{a b \ldots}\left(S_{0}\right)$ & $\Sigma_{i j \ldots .}^{a b}\left(S_{1}\right)$ & $\Sigma_{i j \ldots}^{a b \ldots}\left(T_{1}\right)$ \\
\hline $2 \mathrm{~T}-\mathrm{a}$ & 5.51 & 9.85 & 8.45 \\
$2 \mathrm{~T}-\mathrm{b}$ & 6.40 & 11.13 & 8.69 \\
$3 \mathrm{~T}-\mathrm{a}$ & 7.35 & 7.79 & 15.37 \\
$3 \mathrm{~T}-\mathrm{b}$ & 8.75 & 15.66 & 11.32 \\
$3 \mathrm{~T}-\mathrm{c}$ & 12.11 & 7.16 & 4.84 \\
$4 \mathrm{~T}-\mathrm{a}$ & 5.55 & 11.62 & 11.00 \\
$4 \mathrm{~T}-\mathrm{b}$ & 6.51 & 19.10 & 7.97 \\
$4 \mathrm{~T}-\mathrm{c}$ & 3.00 & 12.35 & 9.53 \\
$4 \mathrm{~T}-\mathrm{d}$ & 4.60 & 13.57 & 11.87 \\
$4 \mathrm{~T}-\mathrm{e}$ & 1.41 & 10.11 & 7.03 \\
$4 \mathrm{~T}-\mathrm{f}$ & 8.80 & 18.13 & 14.29 \\
$4 \mathrm{~T}-\mathrm{g}$ & 11.09 & 25.14 & 13.61 \\
$4 \mathrm{~T}-\mathrm{h}$ & 3.73 & 12.41 & 10.92 \\
$4 \mathrm{~T}-\mathrm{i}$ & 9.12 & 16.51 & 14.68 \\
$4 \mathrm{~T}-\mathrm{j}$ & 2.64 & 12.25 & 9.57 \\
\hline
\end{tabular}


8 Difference density plots 

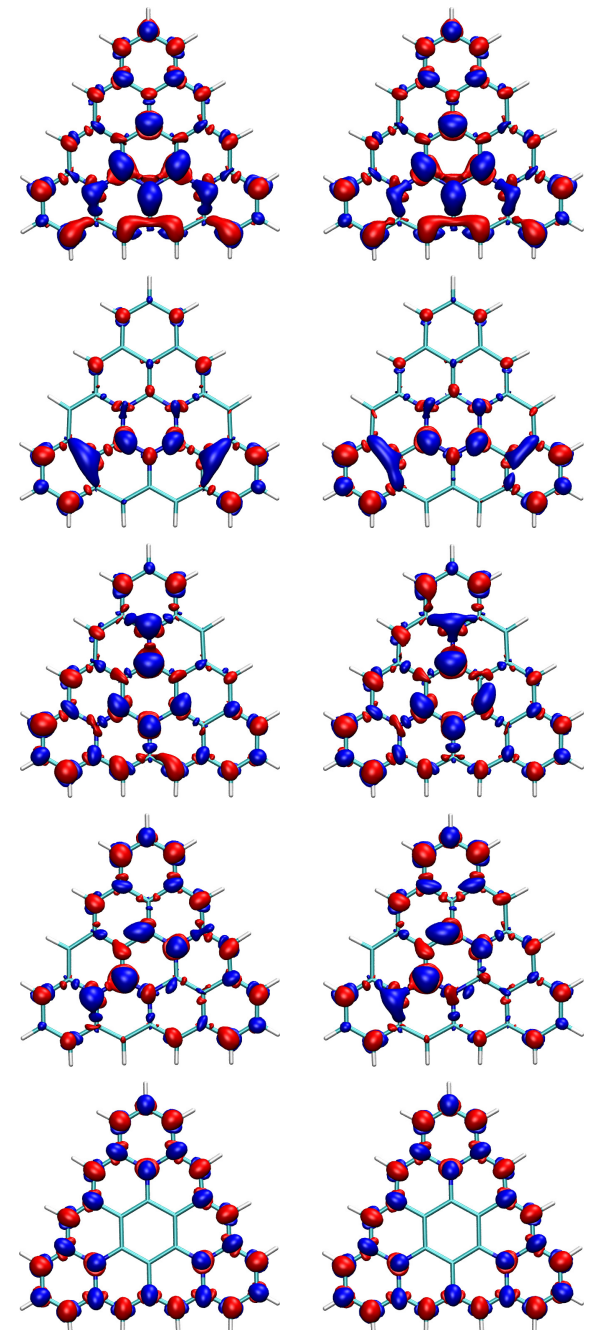

Figure S6: Difference density plots $\left(\sigma=0.002 \mathrm{e} / \mathrm{bohr}^{3}\right)$ associated to the $S_{1} \leftarrow S_{0}$ (left) $T_{1} \leftarrow S_{0}$ (right) excitations, and calculated at the SCSCC2/def2-TZVP level. Red (blue) color indicates decreased (increased) electron density between the ground and excited states. From top to bottom: $4 \mathrm{~T}-\mathrm{a}, 4 \mathrm{~T}-\mathrm{b}, 4 \mathrm{~T}-\mathrm{c}, 4 \mathrm{~T}-\mathrm{d}$, and $4 \mathrm{~T}-\mathrm{e}$. 

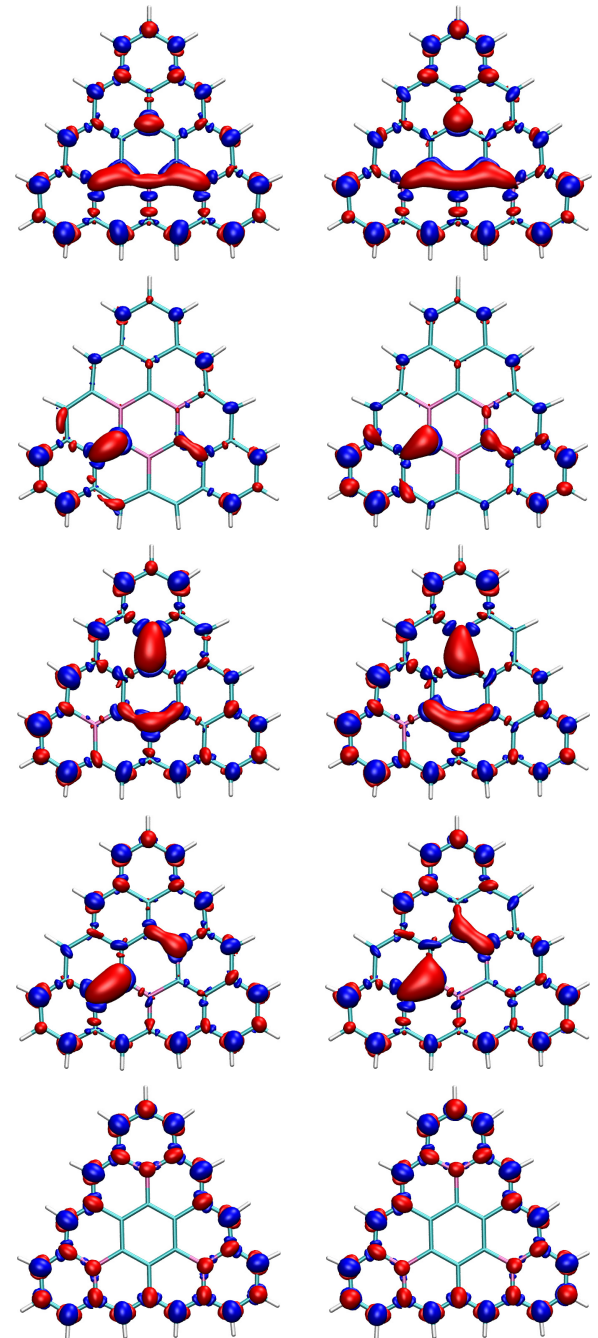

Figure S6 (cont.): Difference density plots $\left(\sigma=0.002 \mathrm{e} / \mathrm{bohr}^{3}\right)$ associated to the $S_{1} \leftarrow S_{0}$ (left) $T_{1} \leftarrow S_{0}$ (right) excitations, and calculated at the SCS-CC2/def2-TZVP level. Red (blue) color indicates decreased (increased) electron density between the ground and excited states. From top to bottom: 4T-f, 4T-g, 4T-h, 4T-i, and 4T-j. 
9 Relationships between $\Delta E_{S T}$ and the CT delocalization volume 


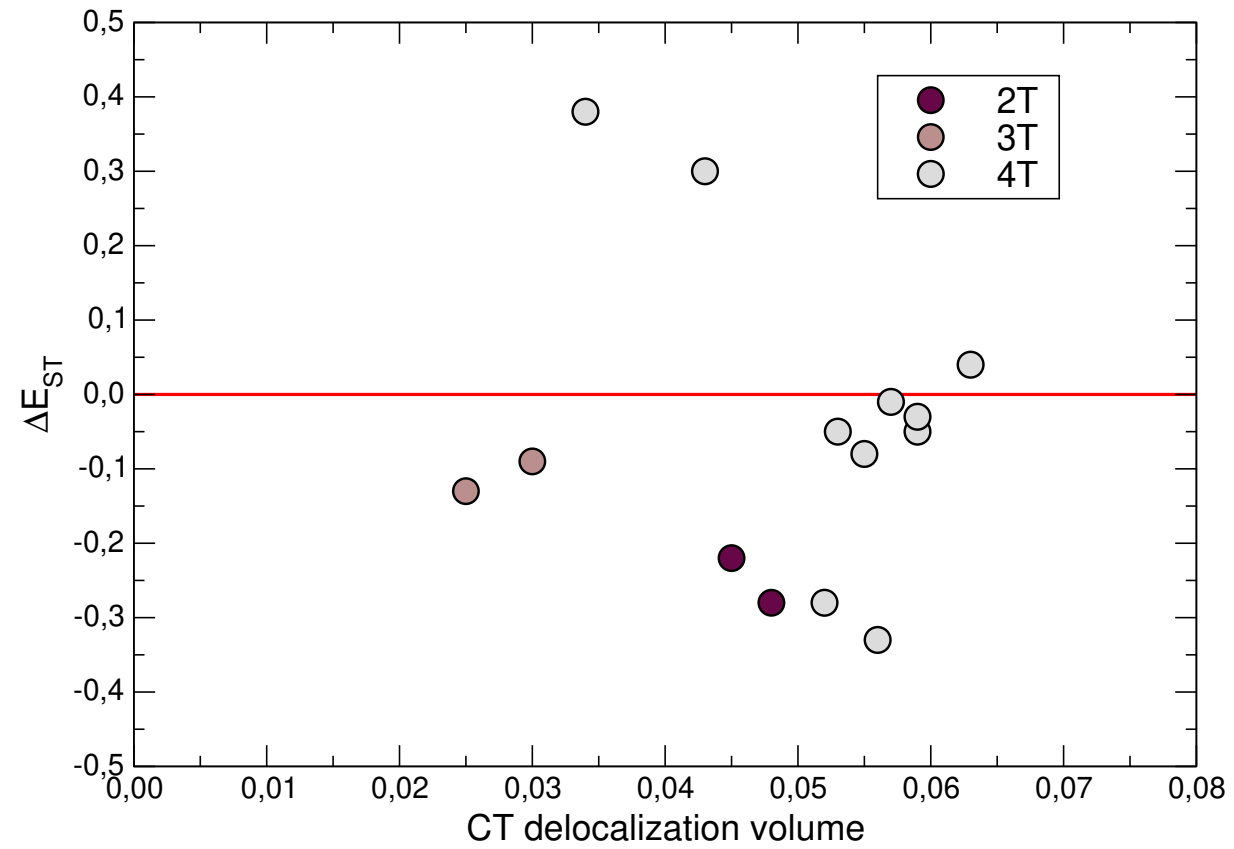

Figure S7: Relationships between $\Delta E_{S T}$ (in eV) and the CT delocalization volume (in $\mathrm{nm}^{3}$ ), all calculated at the SCS-CC2/def2-TZVP level. The red line is a guide to the eye. 


\section{Zero-field splitting and triplet exciton size}

We also calculated the magnitude of the splitting of the triplet sublevels ( $\left.S=1 ; M_{s}=0, \pm 1\right)$ in the absence of any external field, i.e., the ZeroField Splitting (ZFS) interactions, of interest for the Electron Paramagnetic Resonance (EPR) spectra and quantified in term of the absolute values of $D$ and $E:^{4}$

$\hat{H}_{Z F S}=\hat{\mathbf{S}} \cdot \hat{\mathbf{D}} \cdot \hat{\mathbf{S}}=D_{x x} \hat{S}_{x}^{2}+D_{y y} \hat{S}_{y}^{2}+D_{z z} \hat{S}_{z}^{2}=D\left(\hat{S}_{z}^{2}-\frac{1}{3} \hat{\mathbf{S}}^{2}\right)+E\left(\hat{S}_{x}^{2}-\hat{S}_{y}^{2}\right)$,

with $D_{i i}$ the principal values of the ZFS diagonal tensor $\hat{\mathbf{D}}$, which by convention are recasted as:

$$
\begin{array}{r}
D=D_{z z}-\frac{1}{2}\left(D_{x x}+D_{y y}\right), \\
E=\frac{1}{2}\left(D_{x x}-D_{y y}\right) .
\end{array}
$$

Assuming a point-dipole distribution of the unpaired spin for triplet excitedstates, and in absence of spin-orbit and crystal-field contributions to the ZFS in organic systems, ${ }^{5}$ its averaged distance $(\Delta r)$ can be also obtained from the $D$ values and suppose an estimate of the size of the photoexcited triplet exciton: ${ }^{6}$

$$
D=\frac{3}{4} \frac{\mu_{0}}{4 \pi h}\left(g_{e} \mu_{B}\right)^{2}\left\langle\frac{1-3 \cos ^{2} \theta}{\Delta r^{3}}\right\rangle,
$$

with $\Delta r$ the distance between the spins of the unpaired electrons, $\theta$ the angle between the spin vectors and the dipolar $z$-axis, and $\mu_{0}, g_{e}$, and $\mu_{B}$ physical constants.

The Zero-Field-Splitting (ZFS) calculations were performed with the $\omega$ B97X-D functional ${ }^{7}$ and the ORCA 4.0 package, according to recent benchmarking, ${ }^{8}$ and the IGLO-II basis set,${ }^{9}$ a basis set tailored for accurate calcu- 
lation of magnetic and related properties. ${ }^{10}$ The ZFS tensor was always selfconsistently calculated using spin-Unrestricted Natural Orbitals (UNO), ${ }^{11}$ as also recommended in the literature. ${ }^{12}$

We observe much smaller $E$ than $D$ values, see Table S3, with the former magnitude vanishing for perfect axial symmetries (i.e., 2T-a, 2T-b, 4T-e, and $4 \mathrm{~T}-\mathrm{j}$ ) thus confirming their rotational symmetry. $\Delta r$ is calculated to be between 3.3 and $5.7 \AA$ for all the systems, with some general increase with system size going from $2 \mathrm{~T}$ to $4 \mathrm{~T}$. These are reasonable values compared with the estimated exciton size for the triplet (ground-state) of 2,6,10-Tri-tertButyltriangulene, ${ }^{13}$ around $5.6 \AA$, or for the photoexcited triplet state of tetracene and pentacene, ${ }^{14}$ around $3.8 \AA$. For the case of other synthesized TADF emitters with triangulene-derived cores, as DABNA and TABNA, we calculate a value of 4.3 and $3.8 \AA$ for $\Delta r$, respectively, in agreement with previous estimates, ${ }^{15}$ and close to those found here for $2 \mathrm{~T}-4 \mathrm{~T}$; indicating a fast intra-molecular migration of the triplet exciton in all the compounds studied. 
Table S3: Calculated $D$ and $E$ EPR parameters $(D / h c$ and $E / h c$ in $\left.10^{3} \mathrm{~cm}^{-1}\right)$ and exciton size $(\Delta r$, in $\AA)$ at the $\omega$ B97X-D/IGLO-II level.

\begin{tabular}{lrrr}
\hline Compound & $D$ & $E$ & $\Delta r$ \\
\hline $2 \mathrm{~T}-\mathrm{a}$ & 63.78 & 0.03 & 3.44 \\
$2 \mathrm{~T}-\mathrm{b}$ & 58.19 & 0.03 & 3.55 \\
$3 \mathrm{~T}-\mathrm{a}$ & -51.11 & -9.21 & 3.70 \\
$3 \mathrm{~T}-\mathrm{b}$ & -40.10 & -7.83 & 4.02 \\
$3 \mathrm{~T}-\mathrm{c}$ & 18.03 & 0.71 & 5.24 \\
$4 \mathrm{~T}-\mathrm{a}$ & 20.21 & 2.04 & 5.05 \\
$4 \mathrm{~T}-\mathrm{b}$ & 18.84 & 1.89 & 5.17 \\
$4 \mathrm{~T}-\mathrm{c}$ & 22.20 & 1.63 & 4.89 \\
$4 \mathrm{~T}-\mathrm{d}$ & 21.39 & 1.04 & 4.95 \\
$4 \mathrm{~T}-\mathrm{e}$ & 31.56 & 0.00 & 4.35 \\
$4 \mathrm{~T}-\mathrm{f}$ & -35.59 & -2.64 & 4.18 \\
$4 \mathrm{~T}-\mathrm{g}$ & 13.87 & 1.88 & 5.72 \\
$4 \mathrm{~T}-\mathrm{h}$ & 16.79 & 0.50 & 5.37 \\
$4 \mathrm{~T}-\mathrm{i}$ & 17.14 & 1.03 & 5.33 \\
$4 \mathrm{~T}-\mathrm{j}$ & 29.31 & 0.06 & 4.46 \\
\hline
\end{tabular}




\section{References}

[1] Sandoval-Salinas, M. E.; Carreras, A.; Casanova, D. Triangular graphene nanofragments: open-shell character and doping. Physical Chemistry Chemical Physics 2019, 21, 9069-9076.

[2] de Silva, P. Inverted Singlet-Triplet Gaps and Their Relevance to Thermally Activated Delayed Fluorescence. The Journal of Physical Chemistry Letters 2019, 10, 5674-5679.

[3] Leupin, W.; Wirz, J. Low-lying electronically excited states of

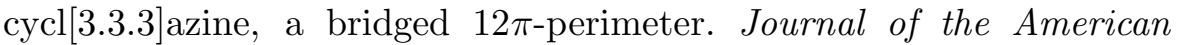
Chemical Society 1980, 102, 6068-6075.

[4] Richert, S.; Tait, C. E.; Timmel, C. R. Delocalisation of photoexcited triplet states probed by transient EPR and hyperfine spectroscopy. Journal of Magnetic Resonance 2017, 280, 103-116.

[5] Loboda, O.; Minaev, B.; Vahtras, O.; Schimmelpfennig, B.; Ågren, H.; Ruud, K.; Jonsson, D. Ab initio calculations of zero-field splitting parameters in linear polyacenes. Chemical Physics 2003, 286, 127-137.

[6] Buta, M. C.; Toader, A. M.; Frecus, B.; Oprea, C. I.; Cimpoesu, F.; Ionita, G. Molecular and Supramolecular Interactions in Systems with Nitroxide-Based Radicals. International Journal of Molecular Sciences 2019, 20, 4733.

[7] Chai, J.-D.; Head-Gordon, M. Long-range corrected hybrid density functionals with damped atom-atom dispersion corrections. Physical Chemistry Chemical Physics 2008, 10, 6615-6620.

[8] Salvitti, G.; Negri, F.; Pérez-Jiménez, Á. J.; San-Fabián, E.; Casanova, D.; Sancho-García, J. C. Investigating the (Poly) Radicaloid 
Nature of Real-World Organic Compounds with DFT-Based Methods. The Journal of Physical Chemistry A 2020, 124, 3590-3600.

[9] Schindler, M.; Kutzelnigg, W. Theory of magnetic susceptibilities and NMR chemical shifts in terms of localized quantities. II. Application to some simple molecules. The Journal of Chemical Physics 1982, 76, 1919-1933.

[10] Jensen, F. Basis set convergence of nuclear magnetic shielding constants calculated by density functional methods. Journal of Chemical Theory and Computation 2008, 4, 719-727.

[11] Neese, F. Calculation of the zero-field splitting tensor on the basis of hybrid density functional and Hartree-Fock theory. The Journal of Chemical Physics 2007, 127, 164112.

[12] Jost, P.; van Wüllen, C. Why spin contamination is a major problem in the calculation of spin-spin coupling in triplet biradicals. Physical Chemistry Chemical Physics 2013, 15, 16426-16427.

[13] Inoue, J.; Fukui, K.; Kubo, T.; Nakazawa, S.; Sato, K.; Shiomi, D.; Morita, Y.; Yamamoto, K.; Takui, T.; Nakasuji, K. The first detection of a Clar's hydrocarbon, 2,6,10-tri-tert-butyltriangulene: a groundstate triplet of non-Kekulé polynuclear benzenoid hydrocarbon. Journal of the American Chemical Society 2001, 123, 12702-12703.

[14] Bayliss, S. L.; Chepelianskii, A. D.; Sepe, A.; Walker, B. J.; Ehrler, B.; Bruzek, M. J.; Anthony, J. E.; Greenham, N. C. Geminate and nongeminate recombination of triplet excitons formed by singlet fission. Physical Review Letters 2014, 112, 238701.

[15] Pershin, A.; Hall, D.; Lemaur, V.; Sancho-García, J.-C.; Muccioli, L.; 
Zysman-Colman, E.; Beljonne, D.; Olivier, Y. Highly emissive excitons with reduced exchange energy in thermally activated delayed fluorescent molecules. Nature Communications 2019, 10, 1-5. 